\title{
Efficacy, Safety and Pharmacokinetics of Up to Two Courses of the Rituximab Biosimilar CT-P10 Versus Innovator Rituximab in Patients with Rheumatoid Arthritis: Results up to Week 72 of a Phase I Randomized Controlled Trial
}

\author{
Dae Hyun Yoo ${ }^{1}$ Chang-Hee $\mathrm{Suh}^{2} \cdot$ Seung Cheol Shim ${ }^{3}$ - Slawomir Jeka ${ }^{4}$. \\ Francisco Fidencio Cons Molina ${ }^{5} \cdot$ Pawel Hrycaj $^{6} \cdot$ Piotr Wiland $^{7}$. \\ Eun Young Lee ${ }^{8} \cdot$ Francisco G. Medina-Rodriguez $^{9} \cdot$ Pavel Shesternya $^{10}$. \\ Sebastiao Radominski ${ }^{11}$ - Marina Stanislav ${ }^{12}$. Volodymyr Kovalenko ${ }^{13}$. \\ Dong Hyuk Sheen ${ }^{14} \cdot$ Leysan Myasoutova $^{15} \cdot$ Mie Jin Lim ${ }^{16} \cdot$ Jung-Yoon Choe $^{17}$. \\ Sang Joon Lee ${ }^{18} \cdot$ Sung Young Lee ${ }^{18} \cdot$ Sung Hwan Kim ${ }^{18} \cdot$ Won Park $^{16}$
}

(C) The Author(s) 2017. This article is an open access publication

\begin{abstract}
Background CT-P10 is a biosimilar of innovator rituximab (RTX), a biological therapy used to treat patients with rheumatoid arthritis (RA) who have responded inadequately to anti-tumor necrosis factor agents.

Objective Our objective was to compare the clinical profile of CT-P10 versus RTX in patients with RA who received up to two courses of treatment and were followed for up to 72 weeks.

Methods In this multicenter double-blind phase I study, patients were randomized 2:1 to receive CT-P10 $1000 \mathrm{mg}$
\end{abstract}

Electronic supplementary material The online version of this article (doi:10.1007/s40259-017-0232-7) contains supplementary material, which is available to authorized users.

Won Park

parkwon@inha.ac.kr

1 Hanyang University Hospital for Rheumatic Diseases, Seoul, Republic of Korea

2 Ajou University School of Medicine, Suwon, Republic of Korea

3 Chungnam National University Hospital, Daejeon, Republic of Korea

4 Collegium Medicum UMK, University Hospital No. 2, Bydgoszcz, Poland

5 Centro de Investigación en Artritis y Osteoporosis, Mexicali, Mexico

6 Poznań University of Medical Sciences, Poznań, Poland

7 Medical University of Wrocław, Wrocław, Poland

8 Seoul National University College of Medicine, Seoul, Republic of Korea or RTX $1000 \mathrm{mg}$ at weeks 0 and 2. Based on disease activity, patients could receive a second course of treatment between weeks 24 and 48. Efficacy endpoints, including mean change from baseline in Disease Activity Score using 28 joints (DAS28), safety, immunogenicity, pharmacokinetics, and pharmacodynamics were evaluated. Results In total, 154 patients were randomized to CT-P10 or RTX ( $n=103$ and 51, respectively); 137 ( $n=92$ and 45) completed the first course of treatment, of whom 83 $(n=60$ and 23) were re-treated. Improvements from baseline in all efficacy endpoints were highly similar between the CT-P10 and RTX groups over both treatment courses. At week 24 after the second course, mean change

La Salle University, Mexico City, Mexico

10 Krasnoyarsk State Medical University, Krasnoyarsk, Russia

11 Universidade Federal do Paraná, Curitiba, Brazil

12 Research Rheumatology Institute n. a. V.A. Nassonova, Moscow, Russia

13 National Scientific Center, Kiev, Ukraine

14 Eulji University Hospital, Daejeon, Republic of Korea

15 Research Medical Complex Vashe Zdorovie, Kazan, Russia

16 School of Medicine, IN-HA University, Incheon, Republic of Korea

17 School of Medicine, Catholic University of Daegu, Daegu, Republic of Korea

18 CELLTRION, Inc., Incheon, Republic of Korea 
from week 0 of the first course in DAS28 erythrocyte sedimentation rate was -2.47 and -2.04 for CT-P10 and RTX, respectively, $(p=0.1866)$ and in DAS28 C-reactive protein was -2.32 and -2.00 , respectively $(p=0.3268)$. The proportion of patients positive for antidrug antibodies at week 24 after the second treatment course was $20.0 \%$ and $21.7 \%$ in the CT-P10 and RTX groups, respectively. The safety profile of CT-P10 was comparable to that of RTX, and pharmacokinetic and pharmacodynamic properties were similar.

Conclusions In patients with RA, efficacy, safety, and other clinical data were comparable between CT-P10 and RTX after up to two courses of treatment over 72 weeks. (ClinicalTrials.gov identifier NCT01534884).

\section{Key Points}

The efficacy of the rituximab biosimilar CT-P10 and innovator rituximab was comparable after up to two courses of treatment in patients with rheumatoid arthritis.

Pharmacokinetics, pharmacodynamics, immunogenicity, and safety profiles up to week 72 were also similar in the two treatment groups.

\section{Introduction}

Innovator rituximab (RTX) has been approved for use in patients with rheumatoid arthritis (RA) since 2006 [1]. Approval of RTX for use in combination with methotrexate in patients with moderate to severe RA that inadequately responded to anti-tumor necrosis factor (TNF) therapy was preceded by randomized controlled trials (RCTs) in which single courses of RTX plus methotrexate led to significant improvements in disease activity compared with placebo or methotrexate alone $[2,3]$.

Expiration dates for various RTX patents are approaching and therefore interest has arisen in the development of "biosimilar" versions that might reduce the cost of rituximab therapy and hence increase patient accessibility to this important treatment option. CT-P10 is a biosimilar of RTX that has recently been approved in Europe for use in all indications held by RTX [4]. CT-P10 has an identical primary structure to RTX and very similar higher-order properties and quality characteristics. Extensive laboratory comparisons of the two drugs have shown that their biological activities are also highly comparable [5]. A phase I RCT of CT-P10 versus RTX in patients with active RA demonstrated that the pharmacokinetics of the two drugs after a single course of treatment were statistically equivalent, and that their efficacy, pharmacodynamics, immunogenicity, and safety were similar up to week 24 [5]. Many studies have shown that RTX re-treatment maintains long-term therapeutic benefit [6-8] and, as such, there is interest in the repeated use of CT-P10. To this end, and to allow further comparison of CT-P10 and RTX, patients could receive a second course of treatment in the same phase I RCT and were followed thereafter to investigate efficacy, safety, pharmacokinetics, and other clinical data up to week 72 .

\section{Methods}

\subsection{Patients}

The design of this phase I RCT and patient eligibility criteria have previously been described in detail [5]. In summary, participants were aged 18-75 years and had a diagnosis of RA according to the revised 1987 American College of Rheumatology (ACR) classification criteria for $\geq 6$ months. Eligible patients also had active RA as defined by $\geq 6 / 66$ swollen joints and $\geq 6 / 68$ tender joints with serum C-reactive protein $(\mathrm{CRP}) \geq 1.5 \mathrm{mg} / \mathrm{dl}$ or erythrocyte sedimentation rate $(\mathrm{ESR}) \geq 28 \mathrm{~mm} / \mathrm{h}$; experienced an inadequate response or were intolerant to anti-TNF agents; received methotrexate treatment (oral or parenteral 10-25 $\mathrm{mg} /$ week at stable dose) for at least 12 preceding weeks. Exclusion criteria included prior treatment with more than two biologic agents; allergies/hypersensitivity to murine, chimeric, human, or humanized proteins; history of rheumatic autoimmune disease other than RA (except secondary Sjögren's syndrome); and history of significant systemic involvement secondary to RA. All patients provided written informed consent.

\subsection{Study Design and Treatment}

This was a multicenter, randomized, two-arm, parallelgroup, double-blind phase I study (ClinicalTrials.gov identifier: NCT01534884). The study started at day 0 ( $\leq 6$ weeks after screening) and extended to week 72 . The study was un-blinded to predefined study teams at week 24 for analysis of the primary results; however, the investigators, patients, and other study teams remained blinded to treatment until study end. The protocol for the study was approved by regulatory authorities and the ethics committees of all participating centers. The study was conducted in accordance with the Declaration of Helsinki and the International Conference on Harmonization Good Clinical Practice guidelines. 
As previously reported, patients were randomly assigned $2: 1$, using a computer-generated randomization schedule, to CT-P10 (CELLTRION Inc., Incheon, Republic of Korea) or RTX (Roche, Welwyn Garden City, UK) on day 0 [5]. Patients received up to two courses of treatment during the study, with each course comprising two intravenous infusions of CT-P10 $1000 \mathrm{mg}$ or RTX $1000 \mathrm{mg}$ separated by 2 weeks. All patients were administered a first course of treatment on weeks 0 and 2. A second course of treatment could be administered between weeks 24 and 48 . To be eligible for a second course of treatment, it was recommended that patients met the following predefined criteria: (1) Disease Activity Score using 28 joint counts (DAS28) combined with ESR (DAS28-ESR) or CRP (DAS28-CRP) between weeks 24 and 40 showing either no response compared with baseline, as defined by European League Against Rheumatism (EULAR) criteria, or relapse (defined as worsening by $\geq 20 \%$ compared with the best response between weeks 16 and 24); and (2) recovery of B-cell or immunoglobulin (Ig)M levels equal to or higher than the lower limit of normal (LLN) or at least $50 \%$ of the baseline value. However, the final decision for initiation of a second course of treatment was made by the investigator. Patients who did not receive the second course of treatment had an end-of-study visit at week 48 unless they withdrew before this time-point. Patients who received a second infusion were followed for 24 weeks after the second course so that the end-of-study visit was held no later than week 72. Oral or parenteral methotrexate (10-25 mg/week) and oral folic acid ( $\geq 5 \mathrm{mg} /$ week) were co-administered to all patients throughout the study. Methylprednisolone (100 mg), paracetamol (usually 500-1000 mg), and chlorpheniramine (2-4 mg or an equivalent dose of another antihistamine) were given before each CT-P10 or RTX infusion.

\subsection{Objective and Endpoints}

The objective of the current analysis was to compare the efficacy, safety, immunogenicity, pharmacokinetics, and pharmacodynamics between CT-P10 and RTX up to week 72. Efficacy assessments at 8-week intervals included DAS28-ESR and DAS28-CRP, Clinical Disease Activity Index (CDAI), Simplified Disease Activity Index (SDAI), and the proportion of patients with a good or moderate response according to EULAR response criteria based on DAS28-CRP. The proportion of patients achieving a clinical response according to the ACR criteria was assessed at weeks 0 and 24 of the second treatment course. Safety assessments included the monitoring of adverse events (including those of special interest such as infections, hypersensitivity/infusion-related reactions, and malignancies or lymphomas). Serum drug concentrations and antidrug antibodies (ADAs) were assessed using an electrochemiluminescent immunoassay method (MSD, Rockville, MD, USA), as described previously [5]. The limit of detection of the assay used to determine serum drug concentrations was $0.01 \mu \mathrm{g} / \mathrm{ml}$, and the upper and lower limits of quantification were $10 \mu \mathrm{g} / \mathrm{ml}$ and $0.02 \mu \mathrm{g} / \mathrm{ml}$, respectively. Samples above the upper limit of the calibration range were diluted. Dilutional linearity was validated by analyzing three replicate quality controls containing $1000 \mu \mathrm{g} / \mathrm{ml} \mathrm{CT-P10}$ at 1:200, 1:400, 1:1000, 1:2000, and 1:10,000 dilutions. Pharmacokinetic endpoints reported here are the serum concentration immediately before the start of the first infusion of the second course $\left(C_{\min }\right)$, maximum serum concentration after the first infusion of the second course $\left(C_{\max , 1}\right)$, trough serum concentration before the second infusion of the second course $\left(C_{\text {trough }}\right)$, and maximum serum concentration after the second infusion of the second course $\left(C_{\max , 2}\right)$. Pharmacodynamic variables assessed were B-cell kinetics, as measured by a FACScalibur flow cytometer (Becton Dickinson and Company, San Jose, CA, USA), serum Ig and CRP levels, and ESR.

\subsection{Statistical Analysis}

All data are summarized using descriptive statistics by treatment group and study visit. For efficacy, immunogenicity, pharmacokinetic, and pharmacodynamic assessments, data beyond week 24 of the study are reported for patients who underwent a second course of treatment only. The change in DAS28 from baseline was compared between treatment groups using a Student's $t$-test and 95\% confidence interval $(\mathrm{CI})$, with a $p$ value $<0.05$ indicating a statistically significant difference between groups. Analysis of covariance (ANCOVA) using the baseline observation carried forward (BOCF) approach for missing data was used in post hoc sensitivity analyses of DAS28 up to week 48 after the first course of treatment. The Kaplan-Meier method with log-rank test was used in a post hoc analysis to compare the time to re-treatment in patients who received a second course of treatment of CT-P10 or RTX. Safety data are reported for all patients regardless of whether they underwent a second course of treatment.

\section{Results}

\subsection{Patient Disposition and Baseline Characteristics}

Patient disposition is summarized in Fig. 1. Briefly, 154 patients were randomly assigned to CT-P10 $(n=103)$ or RTX $(n=51)$. In total, 137 patients $(89.0 \%)$ completed the first course of treatment (92 [89.3\%] and 45 [88.2\%] in the CT-P10 and RTX groups, respectively). 
Fig. 1 Patient disposition. DAS28 Disease Activity Score using 28 joints, EULAR European League Against Rheumatism, $R T X$ innovator rituximab

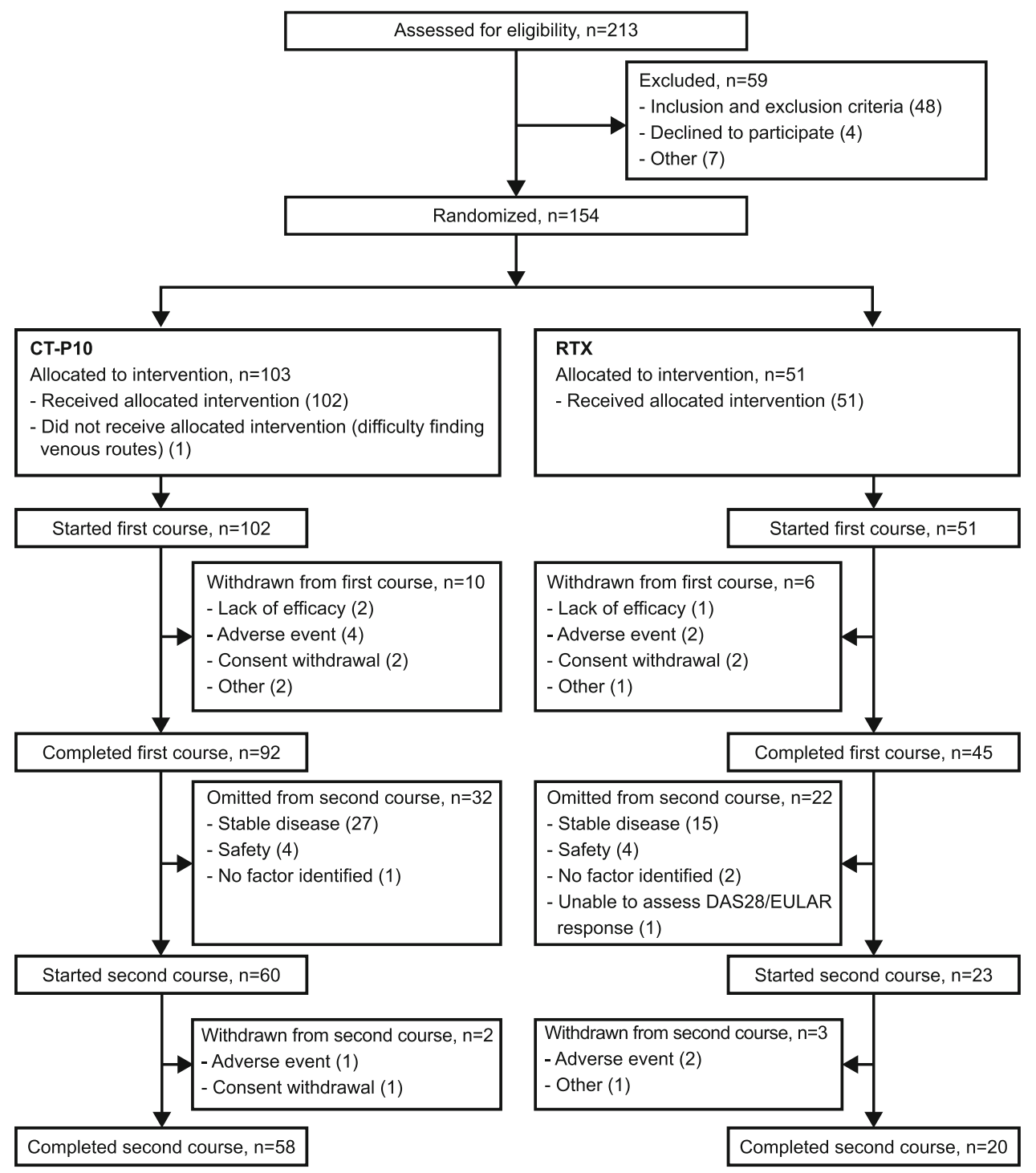

The same proportion of patients in both treatment groups (CT-P10, 66/102 [64.7\%]; RTX, 33/51 [64.7\%]) were eligible for a second course of treatment (i.e., had no response or worsening disease activity after the first course and adequately recovered B-cell or IgM levels). A greater proportion of patients in the CT-P10 group initiated a second course of treatment within 48 weeks of the first course compared with the RTX group; however, this difference was not significant $(58.3 \%[n=60]$ vs. $45.1 \%$ $[n=23$ ]; $p=0.1233$ [Fisher's exact test]). The higher retreatment rate observed in the CT-P10 group was found to be a result of differences in the (protocol-allowable) final decisions of the investigators as to whether or not to initiate a second course in study patients. The most common reasons for eligible patients not being re-treated were maintenance of a good response according to the Visual Analogue Score (VAS)/Health Assessment Questionnaire (HAQ) pain scale (CT-P10, 8 [7.8\%]; RTX, 5 [9.8\%]) and safety concerns (i.e., infusion-related reaction or infection during the previous course; CT-P10, 2 [2.0\%]; RTX, 3 [5.9\%]); these reasons were reported in similar proportions across the two treatment groups. In addition, Kaplan-Meier comparative analysis showed there was no significant difference (log-rank test) between the two treatment groups in either (1) the time to re-treatment in patients who received a second course (median time in weeks [95\% CI]: CT-P10, 46.9 [43.0, 48.1] vs. RTX, 48.3 [44.7, not applicable; NA]; $p=0.2044$ ) or (2) the time to eligibility for re-treatment in all eligible patients regardless of whether re-treatment was given (median time in weeks [95\% CI]: CT-P10, 40.1 [40.0, 41.4] vs. RTX, 40.1 [36.3, 45.1]; $p=0.6433$ ).

Of the 83 patients who started the second course, 78 completed this treatment (Fig. 1). In total, 12 (11.7\%) and $9(17.6 \%)$ patients in the CT-P10 and RTX groups, respectively, withdrew over the course of the whole study. Adverse events were the most common reason for 
withdrawal (5 [4.9\%] and 4 [7.8\%] in the CT-P10 and RTX groups, respectively).

As reported previously, patient demographics were similar between the two groups (Table 1). Patient demographics, baseline disease activity, and prior use of antiTNF agents were also comparable between the two groups for those entering the second course of treatment, and also for those who received the first course only (Table 1).

\subsection{Efficacy}

For patients who received a second course of treatment, DAS28 improvement prior to administration of this course was similar between the two groups. For instance, at week 0 of the second course, the mean change from baseline (week 0 of first course) in DAS28-ESR was -1.00 and -0.79 in the CT-P10 and RTX groups, respectively ( $p=0.4846$ [95\% CI for the difference in change from baseline $-0.79,0.38])$. For DAS28-CRP, the mean change was -0.92 and -0.69 , respectively $(p=0.4343[95 \%$ CI $-0.80,0.35]$ ). Changes in DAS28-ESR, DAS28-CRP, CDAI, and SDAI were comparable between patients treated with CT-P10 and those treated with RTX in the first and the second course (Fig. 2a-d).

At week 24 after the second course of treatment, the mean change from week 0 of the first course in DAS28ESR was -2.47 and -2.04 for CT-P10 and RTX, respectively $(p=0.1866$ [95\% CI $-1.1,0.22]$ ), with similar decreases observed for DAS28-CRP $(-2.32$ and -2.00 in the CT-P10 and RTX groups, respectively; $p=0.3268$ [95\% CI $-0.97,0.33])$. The proportion of patients with a good EULAR-CRP response at week 24 after the second course was comparable between the two treatment groups (Fig. 3). Patients who did not receive the second course had an end-of-study visit at week 48 . The mean change from baseline in DAS28-ESR in patients who did not receive a second course at that time-point (48 weeks after the first course of treatment) was -1.75 and -1.95 in the CT-P10 $(n=30)$ and RTX $(n=18)$ groups, respectively $(p=0.5703[95 \% \mathrm{CI}-0.50,0.90])$. For DAS28-CRP, the mean change was -1.50 and -1.71 in the CT-P10 $(n=30)$ and RTX $(n=18)$ groups, respectively $(p=0.5453[95 \%$ CI $-0.49,0.92])$. Post hoc sensitivity analysis of DAS28-ESR and DAS28-CRP in the safety population using the conservative BOCF imputation method demonstrated comparable responses in the CT-P10 and RTX groups up to week 48 after the first course of treatment. For DAS28-ESR, the mean at baseline and adjusted mean at week 24 and week 48 in the CT-P10 versus RTX groups was 6.80 versus $6.74,4.86$ versus 4.85 , and 6.37 versus 6.10 , respectively. Similar results were also shown for DAS28-CRP (Table 2).
At week 0 of the second course of treatment, the proportions of patients achieving a clinical response according to the ACR20, ACR50, and ACR70 criteria were $33.9 \%$ (20/59), 8.5\% (5/59), and 3.4\% (2/59) in the CT-P10 group, and $21.7 \%(5 / 23), 4.3 \%(1 / 23)$, and 0 in the RTX group, respectively. At week 24 of the second course, ACR20, ACR50, and ACR70 response rates were 69.5\% (41/59), $39.0 \%(23 / 59)$, and $16.9 \%(10 / 59)$ in the CT-P10 group and $39.1 \%(9 / 23), 21.7 \%(5 / 23)$, and $4.3 \%(1 / 23)$ in the RTX group, respectively.

\subsection{Safety}

For safety analyses, patients who received only one course of treatment were followed up to week 48. Patients who received a second course were followed for 24 weeks after the first infusion of the second course. Overall, 73 (71.6\%) and $43(84.3 \%)$ patients in the CT-P10 and RTX groups, respectively, experienced at least one adverse event (Table 3). Infusion-related reactions were reported in 20 $(19.6 \%)$ and $10(19.6 \%)$ patients in the CT-P10 and RTX groups, respectively. Infections were observed in 39 $(38.2 \%)$ and $21(41.2 \%)$ patients in the CT-P10 and RTX groups, respectively (Table 3; also see the Electronic Supplementary Material [ESM] 1). Only one malignancy was reported: a patient in the RTX group had a stage 0 cervix carcinoma that was considered unrelated to the study drug. Adverse events leading to treatment discontinuation were reported for $6(5.9 \%)$ and $4(7.8 \%)$ patients in the CT-P10 and RTX groups, respectively. This included two patients $(2.0 \%)$ in the CT-P10 group (and none in the RTX group) who discontinued the study drug because of infusion-related reactions. No other adverse events leading to discontinuation were reported for more than one patient in either treatment group.

The proportion of patients who experienced at least one serious adverse event was the same in both groups (13.7\%; 14 and 7 patients in the CT-P10 and RTX groups, respectively). Treatment-related serious adverse events occurred in three (2.9\%) patients in the CT-P10 group, with one case each of infusion-related reaction, osteonecrosis, and pruritic rash, and in two (3.9\%) patients in the RTX group, with one case each of neutropenia and lobar pneumonia. All serious adverse events were resolved with appropriate management. No patients died during the study.

\subsection{Immunogenicity}

Immunogenicity results were highly comparable between the two groups after both the first [5] and the second courses of treatment. At week 24 after the second course of treatment, ADAs were detected in $12(20.0 \%)$ and 5 
Table 1 Baseline demographics and disease characteristics (safety population who started first [5] and second course of treatment ${ }^{\mathrm{a}}$ )

\begin{tabular}{|c|c|c|c|c|c|c|}
\hline \multirow[t]{2}{*}{ Characteristics } & \multicolumn{2}{|l|}{ All patients } & \multicolumn{2}{|c|}{ Received first course only } & \multicolumn{2}{|c|}{$\begin{array}{l}\text { Received first and second } \\
\text { courses }\end{array}$} \\
\hline & $\begin{array}{l}\text { CT-P10 } \\
(n=102)\end{array}$ & $\begin{array}{l}\text { RTX } \\
(n=51)\end{array}$ & $\begin{array}{l}\text { CT-P10 } \\
(n=42)\end{array}$ & $\begin{array}{l}\mathrm{RTX} \\
(n=28)\end{array}$ & $\begin{array}{l}\text { CT-P10 } \\
(n=60)\end{array}$ & $\begin{array}{l}\text { RTX } \\
(n=23)\end{array}$ \\
\hline Age (years) & $49.8 \pm 12.6$ & $51.3 \pm 10.9$ & $48.4 \pm 14.1$ & $53.7 \pm 10.6$ & $50.8 \pm 11.4$ & $48.4 \pm 10.8$ \\
\hline \multicolumn{7}{|l|}{ Sex } \\
\hline Female & $88(86.3)$ & $46(90.2)$ & $36(85.7)$ & $26(92.9)$ & $52(86.7)$ & $20(87.0)$ \\
\hline Male & $14(13.7)$ & $5(9.8)$ & $6(14.3)$ & $2(7.1)$ & $8(13.3)$ & $3(13.0)$ \\
\hline \multicolumn{7}{|l|}{ Ethnicity } \\
\hline Caucasian & 69 (67.6) & $35(68.6)$ & $30(71.4)$ & $18(64.3)$ & $39(65.0)$ & $17(73.9)$ \\
\hline Asian & $15(14.7)$ & $9(17.6)$ & $4(9.5)$ & $5(17.9)$ & $11(18.3)$ & $4(17.4)$ \\
\hline Other & 18 (17.6) & $7(13.7)$ & $8(19.0)$ & $5(17.9)$ & $10(16.7)$ & $2(8.7)$ \\
\hline Height (cm) & $161.9 \pm 8.1$ & $162.1 \pm 8.7$ & $161.0 \pm 7.1$ & $159.6 \pm 7.2$ & $162.5 \pm 8.7$ & $165.0 \pm 9.6$ \\
\hline Weight (kg) & $71.4 \pm 17.7$ & $72.4 \pm 16.0$ & $69.9 \pm 14.8$ & $73.5 \pm 16.8$ & $72.4 \pm 19.5$ & $71.0 \pm 15.2$ \\
\hline Body mass index $\left(\mathrm{kg} / \mathrm{m}^{2}\right)$ & $27.2 \pm 6.0$ & $27.5 \pm 5.5$ & $27.0 \pm 5.6$ & $28.7 \pm 5.6$ & $27.3 \pm 6.3$ & $26.1 \pm 5.0$ \\
\hline Disease duration (years) & $11.0 \pm 7.8$ & $10.3 \pm 9.1$ & $10.3 \pm 6.4$ & $9.9 \pm 9.5$ & $11.6 \pm 8.7$ & $10.7 \pm 8.8$ \\
\hline CRP (mg/dl) & $1.8 \pm 1.7$ & $2.1 \pm 3.0$ & $1.4 \pm 1.7$ & $1.9 \pm 2.9$ & $2.0 \pm 1.7$ & $2.4 \pm 3.2$ \\
\hline $\mathrm{ESR}(\mathrm{mm} / \mathrm{h})$ & $49.5 \pm 24.5$ & $50.1 \pm 26.7$ & $45.0 \pm 20.0$ & $51.3 \pm 28.3$ & $52.7 \pm 26.9$ & $48.6 \pm 25.3$ \\
\hline RF positive & $82(80.4)$ & $40(78.4)$ & $34(81.0)$ & $22(78.6)$ & $48(80.0)$ & $18(78.3)$ \\
\hline Anti-CCP positive & $86(84.3)$ & $43(84.3)$ & $36(85.7)$ & $26(92.9)$ & $50(83.3)$ & $17(73.9)$ \\
\hline Swollen joint count (66 joints assessed) & $16.5 \pm 8.2$ & $14.5 \pm 7.0$ & $13.9 \pm 7.3$ & $14.9 \pm 7.2$ & $18.3 \pm 8.4$ & $13.9 \pm 6.9$ \\
\hline Tender joint count (68 joints assessed) & $27.4 \pm 14.8$ & $27.1 \pm 14.2$ & $25.6 \pm 15.8$ & $24.3 \pm 14.3$ & $28.7 \pm 14.1$ & $30.7 \pm 13.5$ \\
\hline DAS28-CRP & $6.0 \pm 0.9$ & $6.0 \pm 0.9$ & $5.8 \pm 0.9$ & $5.9 \pm 1.0$ & $6.2 \pm 0.8$ & $6.0 \pm 0.7$ \\
\hline DAS28-ESR & $6.8 \pm 0.9$ & $6.7 \pm 0.9$ & $6.6 \pm 0.9$ & $6.7 \pm 0.9$ & $6.9 \pm 0.8$ & $6.8 \pm 0.8$ \\
\hline HAQ-DI score & $1.7 \pm 0.7$ & $1.7 \pm 0.7$ & $1.5 \pm 0.7$ & $1.6 \pm 0.8$ & $1.8 \pm 0.6$ & $1.8 \pm 0.5$ \\
\hline \multicolumn{7}{|l|}{ Prior anti-TNF agents } \\
\hline 1 & $88(86.3)$ & $42(82.4)$ & $37(88.1)$ & $25(89.3)$ & $51(85.0)$ & $17(73.9)$ \\
\hline$\geq 2$ & $14(13.7)$ & $9(17.6)$ & $5(11.9)$ & $3(10.7)$ & $9(15.0)$ & $6(26.1)$ \\
\hline \multicolumn{7}{|l|}{ Prior anti-TNF agent status } \\
\hline Failure & $93(91.2)$ & $47(92.2)$ & $37(88.1)$ & $26(92.9)$ & $56(93.3)$ & $21(91.3)$ \\
\hline Intolerance & $9(8.8)$ & $4(7.8)$ & $5(11.9)$ & $2(7.1)$ & $4(6.7)$ & $2(8.7)$ \\
\hline $\begin{array}{l}\text { Duration of prior TNF-antagonist use } \\
\text { (months) }\end{array}$ & $18.9 \pm 20.3$ & $23.7 \pm 26.7$ & $16.6 \pm 17.7$ & $24.0 \pm 29.6$ & $20.5 \pm 22.0$ & $23.3 \pm 23.4$ \\
\hline \multicolumn{7}{|l|}{ Prior TNF antagonists used ${ }^{\mathrm{b}}$} \\
\hline Adalimumab & $37(36.3)$ & $18(35.3)$ & $16(38.1)$ & $13(46.4)$ & $21(35.0)$ & $5(21.7)$ \\
\hline Certolizumab $^{c}$ & $3(2.9)$ & $2(3.9)$ & $1(2.4)$ & $1(3.6)$ & $2(3.3)$ & $1(4.3)$ \\
\hline Etanercept & $30(29.4)$ & $19(37.3)$ & $13(31.0)$ & $12(42.9)$ & $17(28.3)$ & $7(30.4)$ \\
\hline Golimumab & $12(11.8)$ & $3(5.9)$ & $7(16.7)$ & $1(3.6)$ & $5(8.3)$ & $2(8.7)$ \\
\hline Infliximab & $32(31.4)$ & $19(37.3)$ & $9(21.4)$ & $6(21.4)$ & $23(38.3)$ & $13(56.5)$ \\
\hline Investigational anti-TNF agent $^{\mathrm{d}}$ & $3(2.9)$ & $1(2.0)$ & $1(2.4)$ & 0 & $2(3.3)$ & $1(4.3)$ \\
\hline Weekly dose of MTX at baseline (mg) & $15.4 \pm 4.8$ & $15.7 \pm 4.1$ & $14.4 \pm 4.1$ & $15.1 \pm 3.8$ & $16.3 \pm 5.0$ & $16.4 \pm 4.4$ \\
\hline
\end{tabular}

Data are presented as mean \pm standard deviation or $n(\%)$ unless otherwise indicated

$C C P$ cyclic citrullinated peptide, CRP C-reactive protein, DAS28 Disease Activity Score using 28 joints, ESR erythrocyte sedimentation rate, $H A Q$-DI Health Assessment Questionnaire Disability Index, $M T X$ methotrexate, $R F$ rheumatoid factor, $R T X$ innovator rituximab, $T N F$ tumor necrosis factor

${ }^{a}$ Safety population for each treatment course included all patients who received at least one (full or partial) dose of CT-P10 or RTX during that course. Of these, 83 received a second course of treatment

${ }^{\mathrm{b}}$ Some patients had previously received two anti-TNF agents

${ }^{c}$ Includes certolizumab pegol

${ }^{\mathrm{d}}$ Refers to any investigational anti-TNF agent 

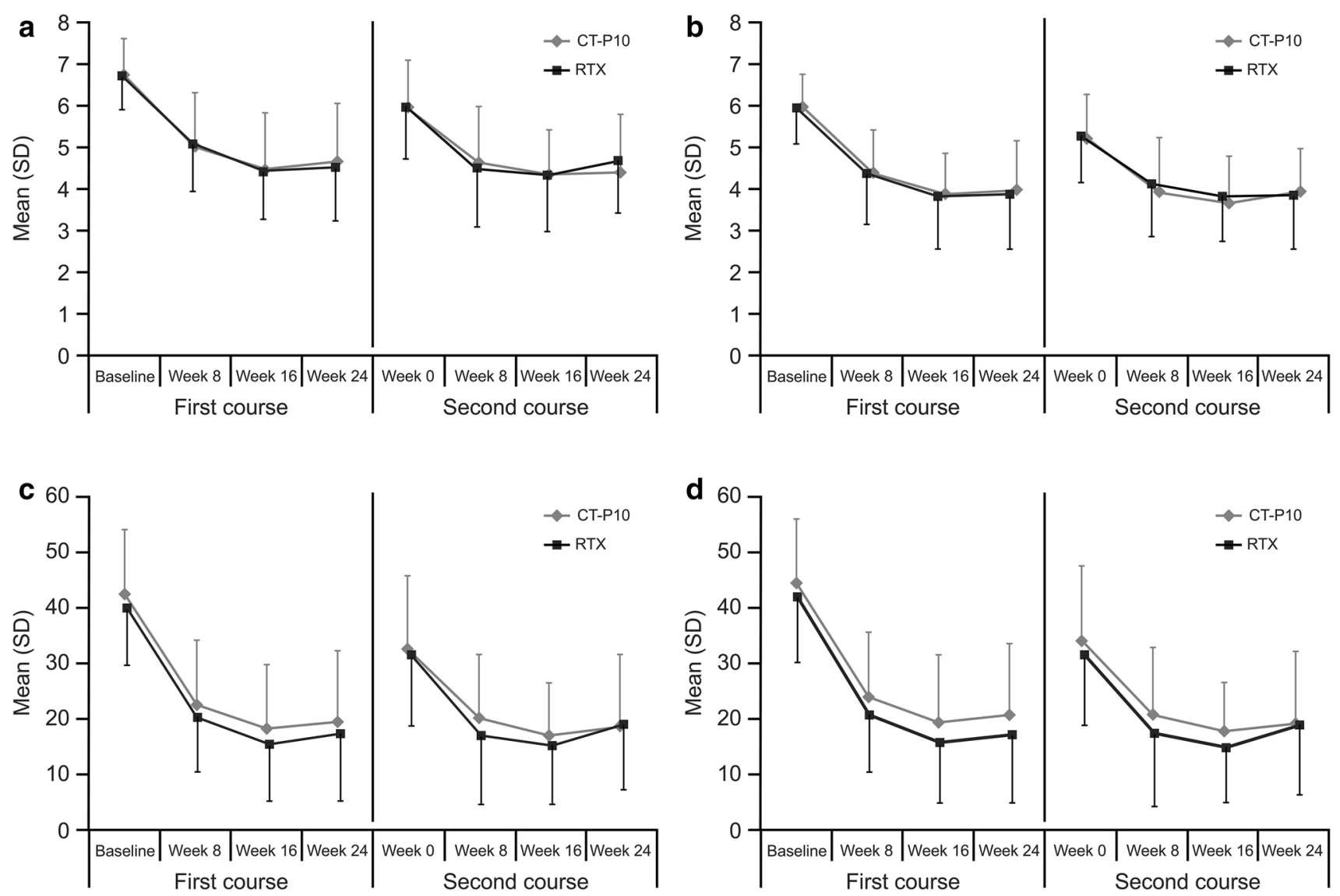

Fig. 2 Disease activity during the first and second courses of treatment as measured by a DAS28-ESR, b DAS28-CRP, c CDAI and d SDAI (efficacy population). Efficacy population for the first course included 100 patients in the CT-P10 and 48 patients in the innovator rituximab treatment groups. Of these, 82 patients received a second course of treatment (59 and 23 patients in the CT-P10 and

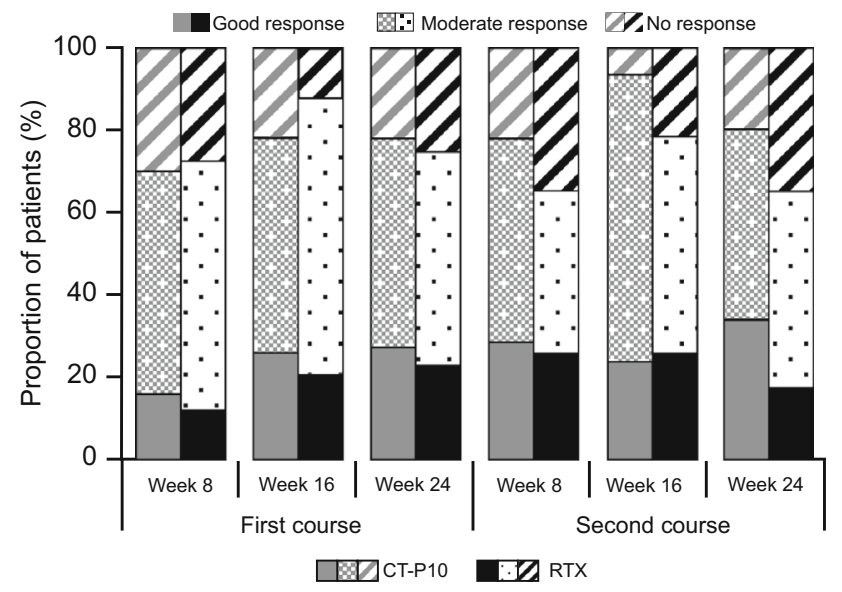

Fig. 3 Response to first and second treatment courses according to European League Against Rheumatism criteria using C-reactive protein (efficacy population). Efficacy population for the first course included 100 patients in the CT-P10 and 48 patients in the innovator rituximab treatment groups. Of these, 82 patients received a second course of treatment (59 and 23 patients in the CT-P10 and innovator rituximab treatment groups, respectively). $R T X$ innovator rituximab innovator rituximab treatment groups, respectively). CDAI Clinical Disease Activity Index, CRP C-reactive protein, DAS28 Disease Activity Score using 28 joints, ESR erythrocyte sedimentation rate, $R T X$ innovator rituximab, SD standard deviation, SDAI Simplified Disease Activity Index

(21.7\%) patients in the CT-P10 and RTX groups, respectively, and neutralizing antibodies were detected in one patient in each group (1.7\% and $4.3 \%$, respectively).

\subsection{Pharmacokinetics and Pharmacodynamics}

As summarized in Table 4, the pharmacokinetics of CTP10 and RTX were similar throughout the second course of treatment. For example, mean \pm standard deviation $C_{\max }$ after the first infusion (i.e., $C_{\max , 1}$ ) was $328.35 \pm 123.02$ and $353.79 \pm 109.35 \mu \mathrm{g} / \mathrm{ml}$ for CT-P10 and RTX, respectively (median $C_{\max , 1}: 352.00$ and $387.00 \mu \mathrm{g} / \mathrm{ml}$, respectively). After the second infusion, $C_{\max }$ (i.e. $C_{\max , 2}$ ) increased to $434.28 \pm 92.28$ and $440.05 \pm 85.62 \mu \mathrm{g} / \mathrm{ml}$, respectively (median $C_{\text {max }, 2}: 435.00$ and $462.00 \mu \mathrm{g} / \mathrm{ml}$, respectively).

Changes in pharmacodynamic parameters were similar between the CT-P10 and RTX groups. In each group, mean CRP and ESR levels decreased from baseline at each timepoint. Median B-cell kinetics were similar between the two 
Table 2 DAS28 up to week 48 after the first course of CT-P10 or innovator ritixumab (safety population ${ }^{\mathrm{a}}$; baseline observation carried forward $^{\mathrm{b}}$ )

\begin{tabular}{lll}
\hline Visit & CT-P10 $(n=102)$ & RTX $(n=51)$ \\
\hline DAS28-ESR & & \\
Baseline & $6.80 \pm 0.86$ & $6.74 \pm 0.85$ \\
Week 24 & $4.86 \pm 0.21$ & $4.85 \pm 0.25$ \\
Week 48 & $6.37 \pm 0.17$ & $6.10 \pm 0.20$ \\
DAS28-CRP & & \\
Baseline & $6.01 \pm 0.90$ & $5.96 \pm 0.88$ \\
Week 24 & $4.12 \pm 0.20$ & $4.09 \pm 0.24$ \\
Week 48 & $5.61 \pm 0.16$ & $5.35 \pm 0.19$ \\
\hline
\end{tabular}

Baseline values are mean $\pm \mathrm{SD}$; values at week 24 and 48 are adjusted mean $\pm \mathrm{SE}$

ANCOVA analysis of covariance, BOCF baseline observation carried forward, CRP C-reactive protein, DAS28 Disease Activity Score using 28 joints, ESR erythrocyte sedimentation rate, $R T X$ innovator rituximab, $S D$ standard deviation, $S E$ standard error

${ }^{a}$ All patients who received at least one (full or partial) dose of CTP10 or RTX

b In this ANCOVA analysis, missing data and data for visits after retreatments were imputed using the conservative BOCF approach

treatment groups (see ESM 2). B-cell counts decreased to below the lower limit of quantification (LLoQ; 20 cells/ $\mu$ l) in each group immediately after the first infusion and remained below this level up to week 32, after which B-cell counts began to recover. Immediately after the first infusion of the second treatment course, counts once again decreased below the LLoQ in all patients and remained below this level in most patients for the duration of the study. However, this B-cell depletion did not appear to impact immunoglobulin levels. At week 0 of the second course, the number of patients with $\operatorname{IgM}, \operatorname{IgA}$, and $\operatorname{IgG}$ levels below the LLN was 1 (1.7\%), 2 (3.3\%), and 0, respectively, in the CT-P10 group and 0,0 , and $1(4.3 \%)$ in the RTX group. Levels were below the LLN on at least one occasion after the start of the second course in 2 (3.3\%), 2 (3.3\%), and $3(5.0 \%)$ patients, respectively, in the CT-P10
Table 4 Pharmacokinetic endpoints during the second course of treatment (pharmacokinetic population ${ }^{\mathrm{a}}$ )

\begin{tabular}{lll}
\hline Endpoint & CT-P10 $(n=57)$ & RTX $(n=21)$ \\
\hline$C_{\text {min }}(\mu \mathrm{g} / \mathrm{ml})$ & & \\
Mean (SD) & $0.08 \pm 0.14$ & $0.03 \pm 0.03$ \\
Median (range) & $0.02(0.02-0.70)$ & $0.02(0.02-0.12)$ \\
$C_{\text {max }, 1}(\mu \mathrm{g} / \mathrm{ml})$ & & \\
Mean $(\mathrm{SD})$ & $328.35 \pm 123.02$ & $353.79 \pm 109.35$ \\
Median (range) & $352.00(25.2-545.0)$ & $387.00(85.6-537.0)$ \\
$C_{\text {trough }}(\mu \mathrm{g} / \mathrm{ml})$ & & \\
Mean (SD) & $75.95 \pm 25.93$ & $80.41 \pm 18.64^{\mathrm{b}}$ \\
Median (range) & $81.30(25.2-132.0)$ & $81.25(46.0-131.0)^{\mathrm{b}}$ \\
$C_{\text {max }, 2}(\mu \mathrm{g} / \mathrm{ml})$ & & \\
Mean (SD) & $434.28 \pm 92.28$ & $440.05 \pm 85.62$ \\
Median (range) & $435.00(228.0-613.0)$ & $462.00(261.0-575.0)$ \\
\end{tabular}

$C_{\max , 1}$ maximum serum concentration after the first infusion of the second course, $C_{\max , 2}$ maximum serum concentration after the second infusion of the second course, $C_{\min }$ serum concentration immediately before the start of the first infusion of the second treatment course, $C_{\text {trough }}$ trough serum concentration before the second infusion of the second course, RTX innovator rituximab, SD standard deviation

a All patients who received a second course of CT-P10 or RTX and provided sufficient blood concentration data

${ }^{\mathrm{b}} n=20$

group and in $1(4.4 \%), 0$, and $1(4.3 \%)$ patients, respectively, in the RTX group. Therefore, most patients were retreated with low B-cell levels but normal IgM levels in accordance with the re-treatment eligibility criteria.

\section{Discussion}

This multinational phase I RCT compared the effects of treatment with CT-P10 and RTX in patients with active RA over a period of up to 72 weeks. We have previously reported that over the first 24 weeks of the study, the pharmacokinetics of CT-P10 and RTX were equivalent and their efficacy, immunogenicity, and pharmacodynamics were highly similar [5]. As reported here, data suggest that
Table 3 Summary of adverse events during the study (safety population $^{\mathrm{a}}$ )

\begin{tabular}{llc}
\hline Adverse events & CT-P10 $(n=102)$ & RTX $(n=51)$ \\
\hline Any adverse event & $73(71.6)$ & $43(84.3)$ \\
Infusion-related reaction & $20(19.6)$ & $10(19.6)$ \\
Infection & $39(38.2)$ & $21(41.2)$ \\
Malignancy & 0 & $1(2.0)$ \\
Study drug discontinuation due to adverse event & $6(5.9)$ & $4(7.8)$ \\
Any serious adverse event & $14(13.7)$ & $7(13.7)$ \\
\hline
\end{tabular}

Data are presented as $n(\%)$

$R T X$ innovator rituximab

${ }^{a}$ All patients who received at least one (full or partial) dose of CT-P10 or RTX 
the biosimilarity of CT-P10 and RTX is continued over an extended period of re-treatment.

Treatments for RA should demonstrate sustained efficacy upon repeated administration, and current guidelines advocate re-treatment as part of the overall disease management strategy [9-12]. Our study was therefore extended beyond week 24 to allow re-treatment based on disease activity. The same proportion of patients in both treatment groups was eligible for a second course of treatment, but more patients in the CT-P10 group than in the RTX group initiated a second course. This was found to be a result of differences in the investigators' final decision to initiate retreatment. As the study was blinded, and following a thorough investigation, no bias during these re-treatment decisions was found.

The effects of a second course of treatment on efficacy endpoints, including DAS28 scores and EULAR response rates, were highly comparable between the CT-P10 and RTX groups. Post hoc sensitivity analysis of DAS28 using the BOCF imputation method up to week 48 after the first course of treatment also demonstrated comparable responses between groups, supporting these primary findings. In this ANCOVA analysis, data for visits after retreatments were considered missing data and imputed with other missing data using the BOCF approach. Decreases in DAS28 scores observed in our study were generally similar to those described after administration of RTX as a single course and after re-treatment in other studies of RA [3, 13-15]. In addition, the proportion of patients with a good or moderate EULAR response in both groups of our study at week 24 after the second treatment course was broadly in line with the proportion of good (15\%) and moderate $(50 \%)$ responders during a single course of treatment in the phase III REFLEX (Randomized Evaluation of Long-term Efficacy of Rituximab in RA) study that first established the superiority of RTX/methotrexate over placebo/methotrexate [3]. Similarly, in the SUNRISE (Study for Understanding Rituximab Safety and Efficacy) trial, which investigated the efficacy and safety of retreatment with RTX, 21 and $48 \%$ of patients achieved a good and moderate EULAR response, respectively, after a second course of treatment [7].

Some numerical differences were observed between the CT-P10 and RTX treatment groups in the proportion of patients achieving a clinical response according to the ACR20, ACR50, and ACR70 criteria at weeks 0 and 24 of the second treatment course. However, it is likely that the small sample sizes, particularly the small number of patients in the RTX group who received a second treatment course, will have had an impact on these response data.

The extended duration of this RCT allowed further comparison of the safety profiles of CT-P10 and RTX in patients with RA. Both drugs displayed an acceptable safety profile, with similar adverse events observed in each treatment group. Once again, safety findings were very much in keeping with data reported in REFLEX [3] and other trials of RTX, including SUNRISE [7]. The mechanism of action of RTX has led to special interest in the frequency of infections following treatment. However, a meta-analysis of RCTs involving the use of RTX to treat RA did not show a significant increase in the rate of serious infections with RTX treatment versus placebo [16], and a global clinical trial program that followed patients for up to 11 years found that the serious infection rate in patients treated with RTX remained stable over time [17]. Overall, $39(38.2 \%)$ and $21(41.2 \%)$ patients in the CT-P10 and RTX groups, respectively, experienced an infection. Notably, data from the AutoImmunity and Rituximab registry have shown that the incidence of infection with RTX in current practice is similar to that in clinical trials [18]. Based on the results of this study, it seems reasonable to assume that the proportion of patients experiencing an infection with repeated exposure to CTP10 will not be different from that observed in the "real world' with RTX.

Infusion-related reactions were observed in $20(19.6 \%)$ and $10(19.6 \%)$ patients in the CT-P10 and RTX groups, respectively, in our study. Infusion-related reactions leading to permanent study discontinuation were reported for $2 / 102(2.0 \%)$ patients in the CT-P10 group and for none in the RTX group. One patient from the CT-P10 group reported a grade 2 infusion-related reaction at the first infusion of the second treatment course but recovered without sequelae and without need for any treatment. Another patient from the CT-P10 group reported a grade 2 infusion-related reaction at the second infusion of the second treatment course but recovered without sequelae after treatment with chlorpheniramine maleate and hydrocortisone. Both of these events were considered non-serious by the investigator.

The proportion of patients with ADAs in this study (18 [17.6\%] and 9 [17.6\%] for CT-P10 and RTX, respectively, at week 24 after the first course [5] and 12 [20.0\%] and 5 [21.7\%], respectively, at week 24 after the second course) was higher than previously reported (12.7\%) [19]. This finding is likely due to the use of a more sensitive ADA assay in our study. The number of patients with neutralizing antibodies was low and similar between groups at week 24 after both the first and the second treatment courses.

Data from the first 24 weeks of this RCT established the pharmacokinetic equivalence of CT-P10 and RTX [5]. Similarity of pharmacokinetics was also evident after the second course of treatment. For example, highly comparable mean $C_{\max }$ values were obtained with CT-P10 and RTX after the second infusion of the second treatment 
course (434.28 and $440.05 \mu \mathrm{g} / \mathrm{ml}$, respectively). Findings for pharmacodynamic parameters, including B-cell counts and serum levels of CRP and ESR, were also similar between the CT-P10 and RTX groups. Decreases in CD20positive cell count observed in both groups of our study are in line with other trials with RTX $[2,3,20]$. Likewise, reductions in CRP and ESR are consistent with the effects of B-cell depletion treatment described in previous studies [21].

Although a number of RTX biosimilars for the treatment of RA are in development [22, 23], CT-P10 is the first to demonstrate biosimilarity to RTX in the clinical setting. Limitations of the current analysis include the relatively small number of patients who received a second course of treatment $(n=83)$ and that not all clinically eligible patients were re-treated. Furthermore, as this trial was powered to formally assess pharmacokinetic equivalence, it was only possible to demonstrate comparable clinical efficacy between the two drugs in this study. However, a recently completed phase III RCT in a larger population of patients with RA indicates that CT-P10 and RTX are also therapeutic equivalents [24].

\section{Conclusions}

This study demonstrated that the treatment of patients with RA with up to two courses of CT-P10 is associated with efficacy, safety, immunogenicity, pharmacokinetics, and pharmacodynamics that are similar to those of its innovator drug, RTX. Based on these data, further long-term comparisons of CT-P10 and RTX in RA are justified.

Acknowledgements The authors wish to thank the patients and study personnel who made this trial possible. They also thank the study investigators. Medical writing support on this manuscript was provided by Alice Wareham $\mathrm{PhD}$ and Rick Flemming PhD, CMPP at Aspire Scientific Limited (Bollington, UK) and funded by CELLTRION, Inc. (Incheon, Republic of Korea).

Study Investigators Germany: Spieler W, Rech HJ, Braun J. Poland: Brzezicki J, Ruzga Z, Piotrowski M, Tlustochowicz W, Porawska W. Mexico: Simon Campos JA, Ramos-Remus C, SalazarParamo M. Russia: Raskina T, Vinogradova I. Brazil: Scheinberg M, Scotton A. Republic of Korea: Park KS, Jun JB, Park YB, Lee SS. Spain: Rey JS, Fernandez-Nebro A, Navarro Blasco FJ. Ukraine: Polyvoda S, Polyakov A.

Author Contributions DHY, SJL, and SYL were involved in the conception and design of the study, acquisition of data and/or analysis and interpretation of data; drafting of the manuscript or revising it critically for important intellectual content; and final approval of the version to be published. SHK was involved in acquisition of data and technical support of the bioanalytical method development and sample analysis; revising the manuscript critically for important intellectual content; and final approval of the version to be published. WP, C-HS, and SCS were involved in the conception and design of the study; drafting of the manuscript or revising it critically for important intellectual content; and final approval of the version to be published. SJ, FFCM, PH, PW, EYL, FGM-R, PS, SR, MS, VK, DHS, LM, MJL, and J-YC were involved in the acquisition of data; drafting of the manuscript or revising it critically for important intellectual content; and final approval of the version to be published. The project management, clinical and medical monitoring, pharmacovigilance, data management, analysis of pharmacokinetic data, biostatistical analysis, and medical writing were performed under contract with PPD, Inc. in collaboration with CELLTRION, Inc. DHY acts as the guarantor for the overall content of this manuscript.

\section{Compliance with Ethical Standards}

Funding This work was funded by CELLTRION, Inc. (Incheon, Republic of Korea). The funding body contributed to the design of the study; the collection, analysis, and interpretation of data; and reviewed drafts and the final version of the manuscript. The final decision to submit the manuscript was taken by the authors.

Conflict of interest DHY is a scientific consultant and on the speaker's bureau of CELLTRION and has received research grants not related to this clinical study. PH and SR received grants from CELLTRION for conducting the clinical trial. MJL received grants from CELLTRION during the conduct of the study. SJL is an employee of, and holds stock options in, CELLTRION. SYL and SHK are employees of CELLTRION. WP received consulting fees during the conduct of the study and grants outside the submitted work from CELLTRION. C-HS, SCS, SJ, FFCM, PW, EYL, FGM-R, PS, MS, VK, DHS, LM, and J-YC have no conflicts of interest.

Ethical approval The study was conducted in accordance with the Declaration of Helsinki and the International Conference on Harmonization Good Clinical Practice guidelines. The protocol for the study was approved by regulatory authorities and the ethics committees of all participating centers, as follows. Brazil: Comitê de Ética em Pesquisa da Associação de Assistência à Criança Deficiente; Comitê de Ética em Pesquisa em Seres Humanos da Universidade Federal de Juiz de Fora; Comitê de Ética em Pesquisa do Hospital de Clínicas da Universidade Federal do Paraná. Germany: Ethics Committee of PPD Germany GmbH \& Co KG. Republic of Korea: Institutional Review Board (IRB) of IN-HA University Hospital; IRB of Hanyang University Medical Center; IRB of Seoul St Mary's Hospital, The Catholic University; SNUMC/SNUH IRB; IRB of Severance Hospital; IRB of Chonnam National University Hospital; IRB of Daegu Catholic University Medical Center; IRB of Daejeon Eulji University Hospital. Mexico: Comitê de Ética ICLE SC; Comitê de Investigación y Ética del Hospital Centro de Especialidades Médicas del Sureste SA de CV; Comitê de Bioetica de la Unidad de Investigación en Enfermedades Cronico Degenerativas; Comitê de Investigación para Estudios en Humanos del "Hospital Médica Sur" S.A.B de C.V; Comitê Bioetico para la Investigación Clínica S.C. IRB. Poland: Bioethics Committee at the Regional Medical Council of Wielkopolska Medical Chamber. Russia: Council on Ethics, Annex to the Order of the Ministry of Healthcare of the Russian Federation. Spain: Comité Etico de Investigación Clínica de Andalucia. Ukraine: Central Commission on Ethics Questions of the MoH of Ukraine.

Informed consent All patients provided written informed consent to participate in the study.

Open Access This article is distributed under the terms of the Creative Commons Attribution-NonCommercial 4.0 International License (http://creativecommons.org/licenses/by-nc/4.0/), which permits any noncommercial use, distribution, and reproduction in any medium, provided you give appropriate credit to the original 
author(s) and the source, provide a link to the Creative Commons license, and indicate if changes were made.

\section{References}

1. Lopez-Olivo MA, Amezaga Urruela M, McGahan L, et al. Rituximab for rheumatoid arthritis. Cochrane Database Syst Rev 2015;(1):CD007356.

2. Edwards JC, Szczepanski L, Szechinski J, et al. Efficacy of B-cell-targeted therapy with rituximab in patients with rheumatoid arthritis. N Engl J Med. 2004;350:2572-81.

3. Cohen SB, Emery P, Greenwald MW, et al. Rituximab for rheumatoid arthritis refractory to anti-tumor necrosis factor therapy: Results of a multicenter, randomized, double-blind, placebo-controlled, phase III trial evaluating primary efficacy and safety at twenty-four weeks. Arthritis Rheum. 2006;54:2793-806.

4. European Medicines Agency. European public assessment report for Truxima. 2017. http://www.ema.europa.eu/docs/en_GB/ document_library/EPAR_-_Summary_for_the_public/human/004112/ WC500222696.pdf. Accessed 27 Mar 2017.

5. Yoo DH, Suh C, Shim SC, et al. A multicentre randomised controlled trial to compare the pharmacokinetics, efficacy and safety of CT-P10 and innovator rituximab in patients with rheumatoid arthritis. Ann Rheum Dis. 2016;76:566-70.

6. Keystone EC, Cohen SB, Emery P, et al. Multiple courses of rituximab produce sustained clinical and radiographic efficacy and safety in patients with rheumatoid arthritis and an inadequate response to 1 or more tumor necrosis factor inhibitors: 5-year data from the REFLEX study. J Rheumatol. 2012;39:2238-46.

7. Mease PJ, Cohen S, Gaylis NB, et al. Efficacy and safety of retreatment in patients with rheumatoid arthritis with previous inadequate response to tumor necrosis factor inhibitors: results from the SUNRISE trial. J Rheumatol. 2010;37:917-27.

8. Wendler J, Burmester GR, Sörensen H, et al. Rituximab in patients with rheumatoid arthritis in routine practice (GERINIS): six-year results from a prospective, multicentre, non-interventional study in 2,484 patients. Arthritis Res Ther. 2014;16:R80.

9. Buch MH, Smolen JS, Betteridge N, et al. Updated consensus statement on the use of rituximab in patients with rheumatoid arthritis. Ann Rheum Dis. 2011;70:909-20.

10. Bukhari M, Abernethy R, Deighton C, et al. BSR and BHPR guidelines on the use of rituximab in rheumatoid arthritis. Rheumatology (Oxford). 2011;50:2311-3.

11. Dudler J, Finckh A, Kyburz D, et al. Swiss consensus statement: Recommendations for optimising re-treatment with MabThera (rituximab) in rheumatoid arthritis. Swiss Med Wkly. 2010;140:w13073.
12. Leandro MJ, Becerra-Fernandez E. B-cell therapies in established rheumatoid arthritis. Best Pract Res Clin Rheumatol. 2011;25:535-48.

13. Mariette X, Rouanet S, Sibilia J, et al. Evaluation of low-dose rituximab for the retreatment of patients with active rheumatoid arthritis: a non-inferiority randomised controlled trial. Ann Rheum Dis. 2014;73:1508-14.

14. Emery P, Deodhar A, Rigby WF, et al. Efficacy and safety of different doses and retreatment of rituximab: a randomised, placebo-controlled trial in patients who are biological naive with active rheumatoid arthritis and an inadequate response to methotrexate (Study Evaluating Rituximab's Efficacy in MTX iNadequate rEsponders (SERENE)). Ann Rheum Dis. 2010;69:1629-35.

15. Tak PP, Rigby WF, Rubbert-Roth A, et al. Inhibition of joint damage and improved clinical outcomes with rituximab plus methotrexate in early active rheumatoid arthritis: the IMAGE trial. Ann Rheum Dis. 2011;70:39-46.

16. Salliot C, Dougados M, Gossec L. Risk of serious infections during rituximab, abatacept and anakinra treatments for rheumatoid arthritis: meta-analyses of randomised placebo-controlled trials. Ann Rheum Dis. 2009;68:25-32.

17. van Vollenhoven RF, Fleischmann RM, Furst DE, et al. Longterm safety of rituximab: final report of the rheumatoid arthritis global clinical trial program over 11 years. J Rheumatol. 2015;42:1761-6.

18. Gottenberg JE, Ravaud P, Bardin T, et al. Risk factors for severe infections in patients with rheumatoid arthritis treated with rituximab in the autoimmunity and rituximab registry. Arthritis Rheum. 2010;62:2625-32.

19. European Medicines Agency. Rituximab. Summary of product characteristics. 2015. http://www.ema.europa.eu/docs/en_GB/ document_library/EPAR_-_Product_Information/human/000165/ WC500025821.pdf. Accessed 21 Feb 2017.

20. Conti F, Ceccarelli F, Massaro L, et al. Biological therapies in rheumatic diseases. Clin Ter. 2013;164:e413-28.

21. Tsiakalos AP, Avgoustidis NK, Moutsopoulos HM. Rituximab therapy in Greek patients with rheumatoid arthritis. Biologics. 2008;2:911-6.

22. da Silva A, Kronthaler U, Koppenburg V, et al. Target-directed development and preclinical characterization of the proposed biosimilar rituximab GP2013. Leuk Lymphoma. 2014;55:1609-17.

23. Vital EM, Kay J, Emery P. Rituximab biosimilars. Expert Opin Biol Ther. 2013;13:1049-62.

24. Yoo D-H, Majstorovic L, Kasay A, et al. Efficacy and safety of CT-P10, rituximab biosimilar candidate, and innovator rituximab in patients with rheumatoid arthritis: results from Phase 3 randomized controlled trial over 24 weeks. Arthritis Rheumatol. 2016;68(Suppl 10):1635. 